\title{
PACKING DEFECTS, CAVITIES, VOLUME FLUCTUATIONS, AND ACCESS TO THE INTERIOR OF PROTEINS. INCLUDING SOME GENERAL COMMENTS ON SURFACE AREA AND PROTEIN STRUCTURE.
}

\author{
by
}

\author{
FREDERIC M. RICHARDS
}

Department of Molecular Biophysics and Biochemistry,

Yale University, Box 1937 Yale Station. New Haven, Connecticut 06520, U.S.A.

\begin{abstract}
Keywords: Secondary structure, tertiary structure, protein dynamics, hydrogen isotope exchange
\end{abstract}

Presented as the 5th Linderstrøm-LANG Lecture at the Carlsberg Laboratory, Copenhagen, on 29 November 1978

\begin{abstract}
A brief review is given of the use of molecular surface area in estimations of hydrophobic forces and of their influence on protein structure. Molecular area can be used as an estimate of the free energy of transfer of a solute between solvents of differing polarity. Area changes that occur on forming secondary and tertiary structural units are examined including the possible uses of such estimates in algorithms for folding peptide chains.

In 1975 LUMRY and ROSENBERG introduced the concept of mobile defects in discussing protein dynamics with particular reference to hydrogen exchange experiments (25). This idea is examined quantitatively in this paper. A first order approach is taken by assuming volume changes to occur by isometric expansion and contraction of the small cavities that occur as packing defects in the structures of all proteins. The positions and mean volume of these defects can be derived from the known X-ray structure using the Voronoi construction. The volume fluctuations of these cavities are assumed to follow a normal distribution leading to a probability of expansion to any preset value, $v_{0}$. The standard deviation of the fluctuation, $\sigma$, is related to the mean isothermal compressibility of the protein and is a characteristic of that particular molecule (8). The parameter $\mathrm{v}_{0}$ is related to the process being considered and should be the same for all proteins. For hydrogen exchange it should be related to the volume of a water molecule. For fluorescence quenching it would reflect the molecular volume of the quencher. The theory has been applied to myoglobin and pancreatic trypsin inhibitor. Reasonable agreement with hydrogen exchange data for the slowly exhanging amide protons can be obtained, but there is difficulty with the rapidly exchanging protons and access to the protein surface. No explicit account has yet been taken of the changes in exchange rate due to primary or secondary structure, factors which will particularly effect the surface positions.
\end{abstract}




\section{INTRODUCTION}

It is a great privilege and honor for me to be invited to address the members of the Carlsberg Laboratory on this day in memory of $\mathrm{KAJ}$ LINDERSTRøM-LANG. The year that I spent with him in the older version of these elegant quarters was the high point of my scientific experience.

At that time, 1954, LANG was immersed in his studies of the proteolytic cleavage of protein substrates and of deuterium-hydrogen exchange of native and denatured proteins, the latter a technique he had recently invented. As part of this work he was intimately concerned with the folding and unfolding of proteins and with both the limiting structures and dynamics of these processes. From our vantage point in the late '70s it is hard to remember that the peptide hypothesis had only recently vanquished all other opposition, that only one full protein sequence was known, that the detailed threedimensional structure of no protein had yet been established and that the proper refolding of a completely unfolded peptide chain had yet to be recognized and demonstrated. The "sausage" model of myoglobin was published only in 1959 , the year of LANG's death. In spite of this lack of a definitive experimental base, LANG gave a very clear and explicit description of what a protein should look like, and he defined the hierarchy of primary, secondary and tertiary structure (24). These concepts and terms are now part of our everyday language in structural molecular biology.

Today I would like to give a brief overview of the work of some of my colleagues and myself over the last few years related to general problems of protein structure and then to concentrate on some estimates of fluctuations in structure which can be related to hydrogen exchange data.

\subsection{Area of the solute-solvent interface}

The structural models of proteins derived from X-ray diffraction studies serve to emphasize the great complexity of these three-dimensional objects. Extraction of generalities from this mass of data has required simplification and idealization of the actual structures. At one level, the topology of the peptide chain can be examined in a search for structural motifs at a level intermediate between secondary and tertiary structure. This has been a very fruitful area in the last few years and has been reviewed in detail very recently by JANE RichaRdSON (37). From a different point of view one can disregard the chain aspect of the structure and concentrate on such geometrical functions as area and volume. Much of the recent work of this type has been reviewed by RichaRDS (34).

One procedure for calculating the area of a protein molecule from the X-ray atomic coordinates was proposed by LEE and RiCHARDs (22). The procedure can be applied to any structure, real or hypothetical, for which a set of Cartesian coordinates can be supplied. The chemical nature of the surface can also be specified, for example non-polar hydrocarbon areas versus polar areas containing oxygen or nitrogen atoms. The first clear-cut result of these calculations was that the surface of small water soluble proteins is about half polar and half non-polar. In retrospect, this result is demanded by the size and structure of the amino acid residues themselves. Since there are no residues which are entirely polar, the surface must have a stong non-polar component regardless of composition or sequence. The difference between associating and non-associating proteins will depend on the surface distribution of the polar and non-polar areas and this, of course, will be sequence dependent. Since there are residues whose side chains are totally nonpolar, appropriate sequences can lead to segments that might be expected to be lipid soluble and thus suitable for association with the nonpolar parts of membranes as well as with each other. For all residues it must be remembered that each has the nitrogen and carbonyl oxygen atoms of the main chain and that these are highly polar groups. The formation of the protein interior will inevitably bury such groups. The effect of their polarity is, at least partially, alleviated by the extensive main chain hydrogen bonding in the units of secondary structure.

A very large area change occurs on going from a hypothetical extended chain to the native protein structure. Much work by HermanN (17), Reynolds, Gilbert and Tanford (32), Chothia (4), and others has made a very plausible case for the use of non-polar solute-solvent interfacial area as a linear measure of the free energy change that occurs on shifting the solute from water to a 
less polar solvent. When the numbers derived from small molecule data are applied to a peptide chain of any appreciable length, the driving force to form a compact, low contact area, structure is seen to be very large. During the folding process for proteins the size of ribonuclease or myoglobin, the area decreases by a factor of about 3 (22). The tens of thousands of square Ångström units involved reflect a hydrophobic contribution to the folding process of several hundred kilocalories.

In a hypothetical folding pathway, one can consider the association of ribonuclease SPeptide and S-Protein, for example, to be divided into two steps: 1) The conversion of the open chain peptide to the conformation that it will have in the complex. (This conformation includes 2 turns of $\alpha$-helix.) 2) The association of this preformed peptide with S-Protein to yield ribonuclease-S. Of the total area change for the peptide in this process, about one-half occurs in step 1 , the formation of the secondary structure and the other half in forming the tertiary structure of the final complex (35).

This roughly equal division of the area change, and by implication of the free energy change, between the formation of the secondary and tertiary structures appears to be quite general. The secondary structural units thus have substantial stability in the absence of the full tertiary structure. If one assumes that the folding of a peptide chain involves the sequential formation of secondary and tertiary structure, then the initially formed secondary units are likely to persist in the final tertiary product. If they do not, then a misfolded intermediate is likely to have enough stability to be detected and represent a substantial slowing of the overall process as the secondary structural reorganization occurs. (See the review of experimental data on this process by CREIGHTON (10).)

As a logical extension of these ideas, Richards and Richmond (36) have suggested that the rank ordering of the amino acids in their tendency to form either $\alpha$-helices or $\beta$-sheets (see for example, CHOU and FASMan, (6)) can be related to the area changes calculated for the formation of the two different types of structure. The correlation for the $\beta$-sheet calculations is particularly good, but the $\alpha$-helix calculations indicate that, although the area changes are important, other factors must play a major role.

In forming the tertiary structure of myoglobin from preformed, connected, helical segments, the area change on associating the separate helixhelix pairs represents a major fraction of the total change for the whole process. Richmond has examined the individual interaction sites in detail and has proposed an algorithm for estimating the probability of any particular helix-helix interaction (38). Using this algorithm, COHEN has produced a program which folds up the 'string and sausage' model of myoglobin into a compact structure (7). The Richmond algorithm limits the number of potential interaction sites on each helix. The Cohen algorithm examines all possible pairings and checks only for acceptable connection length between the helix ends and for steric overlap in the final structure. This procedure reduces over $10^{8}$ pairings to less than 100 possible structures and indicates that these simple geometrical considerations may play a useful part in a more general chain folding algorithm.

Other examples of the application of the concept of solvent accessibility are: the prediction of the location of $\beta$-turns (39); and the prediction of the $\mathrm{pK}$ values of ionizable groups (see discussion and references to the work of GURD and colleagues in (16). The latter studies are a most interesting improvement on the original smeared charge model introduced more than five decades ago by LiNDERSTRøM-LANG (23).

\subsection{Volumes and packing}

Questions of a different sort can be asked about the packing of the atoms in proteins and how this compares with what is known about small molecules. The Voronoi procedure (described below) is a geometrical construction which allows an objective assigments of volume to each atom in a structure. The number so derived can be used as an estimate of the volume occupied by the atom. The volume inside the van der Waals envelope of the atom may be taken as the minimum possible atom volume (in the hard sphere approximation). The ratio of this value to the Voronoi volume is defined as the packing density and is a measure of packing efficiency. The mean value found for the interior of proteins is about equal to that for molecular crystals of 
small organic substances in general and for the free amino acids and small peptides in particular $(33,14,15,5)$. Very seldom is there space for a water molecule in the interior and such molecules are only rarely identified in the electron density maps. It appears highly unlikely that such excellent packing is accidental and would suggest that packing requirements are an important part of the process for selecting between various possible low area conformations of a peptide chain. The native structure of most proteins probably represents the minimum surface conformation compatible with a packing density of about 0.75 .

The above statements refer to averages over the whole protein. The packing density on a smaller scale does vary considerably from region to region in a protein and this variation will now be examined in more detail.

\subsection{Packing defects and volume fluctuations}

LUMRY and ROSENBERG (25) have introduced to the protein structure field the concept of mobile defects, volumes of empty space which migrate through the molecule. The genesis of this proposal lay in the observed hydrogen exchange data then available on proteins and the surprising fluorescence quenching data of LAKOWICZ and WeBER (21) and EFTINK and GHIRON $(11,12)$. The following section presents a quantitative investigation of the first order solution of their hypothesis. A similar concept of fluctuations related to the mechanism of enzyme action has been proposed independently by CARERI (3).

The X-ray model of a protein is normally presented as a static structure, but it is well recognized that this is the time and space average of a variety of instantaneous structures which are rapidly interconverting as a result of thermal motion. This behavior is reflected in the temperature factor, B, which differs, in principle, for each atom and which is a parameter derived directly from the X-ray data. There are many contributions to B such as systematic errors in the data and statistical disorder which are not directly related to motion, but in a careful study these can be minimized and corrected. Thermal motion in most cases is a major component. In protein structure studies, there is normally not enough data to permit more than the estimate of an isotropic B factor although the real motions are obviously much more complex. The thermal component of $B$ is directly proportional to the mean square amplitude of the atomic vibration. A value of 20 (within the range observed in proteins) corresponds to a root mean square displacement of $0.5 \AA$. This type of analysis assumes a gaussian distribution and thus a displacement of $1.5 \AA$ would correspond to a $3 \sigma$ deviation from the mean position. Two such atoms, in contact but not covalently connected, could move in opposite directions and create a cavity $3 \AA$ in diameter with a low but perfectly finite probability.

COOper (8) has made a provocative analysis of fluctuations in small systems based on statistical thermodynamic arguments. The internal energy changes are related to the heat capacity at constant volume and correspond to an r.m.s. fluctuation of about $32 \mathrm{kcal} \cdot \mathrm{mole}^{-1}$ for a molecule of the size of myoglobin near room temperature. Volume fluctuations are related to

Figure 1. The Voroni construction in two dimensions.

a) The black dots are an arbitrary set of points. The medium thickness lines are the interpoint vectors. The light lines are the perpendicular bisectors of the interpoint vectors. The thick lines are the edges of the limiting polygons around each of three points identified by the small concentric circle around the point. These polygons are uniquely defined and yield an area (volume in 3 dimensions) to be assigned to each point. The point on the left is at the surface of the collection. The polygon is not closed and thus the area for this point is undefined in this example.

b) A section through the cubic lattice showing some atoms of the protein and circles indicating the van der Waals envelopes surrouding the nuclei. The crosses mark some of the cube centers. Those labeled a are inside the van der Waals radius of a protein atom. Those labeled $b$ are outside of any protein atom and serve to define a packing defect. The Voroni construction for the defect volumes is modified so that planes perpendicular to vectors from cube centers to protein atoms are drawn tangent to the van der Waals envelope rather than as bisectors. The Voroni polygon for one defect in this section is outlined by the thick lines. 
F. M. RICHARDS: Packing defects and the interior of proteins

a. NORMAL VORONOI CONSTRUCTION

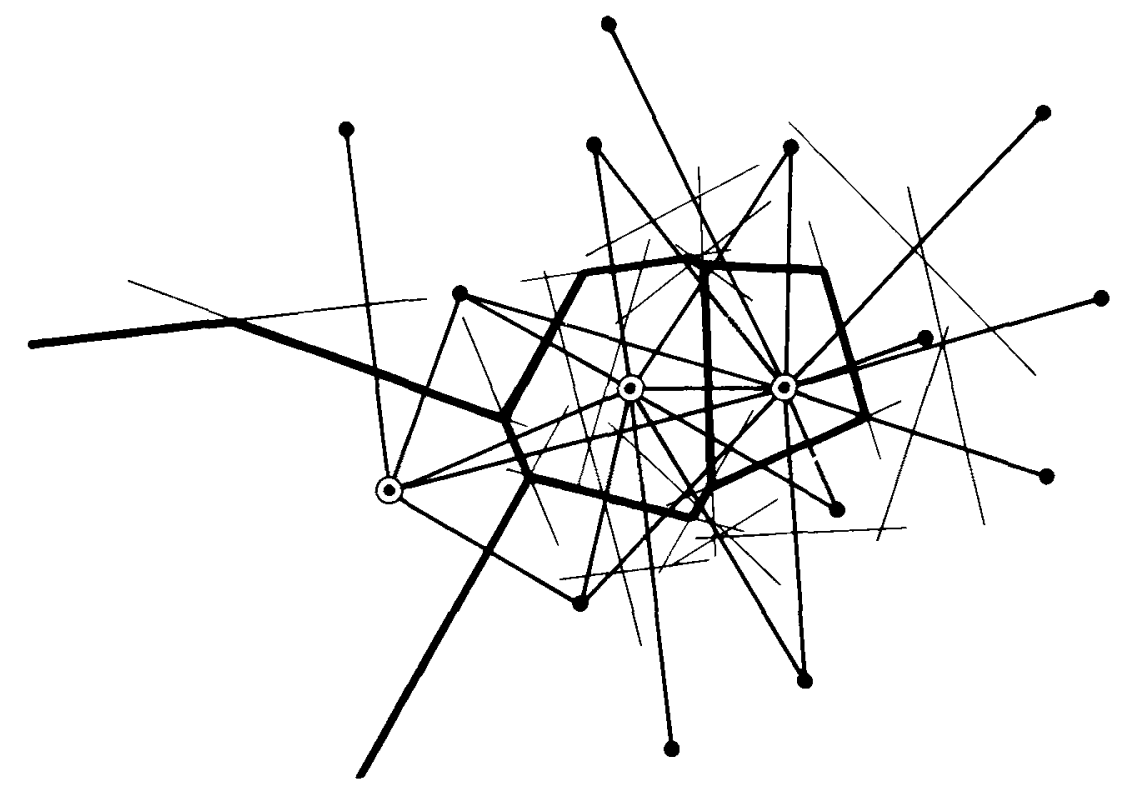

b. VOLUME CONSTRUCTION FOR DEFECTS

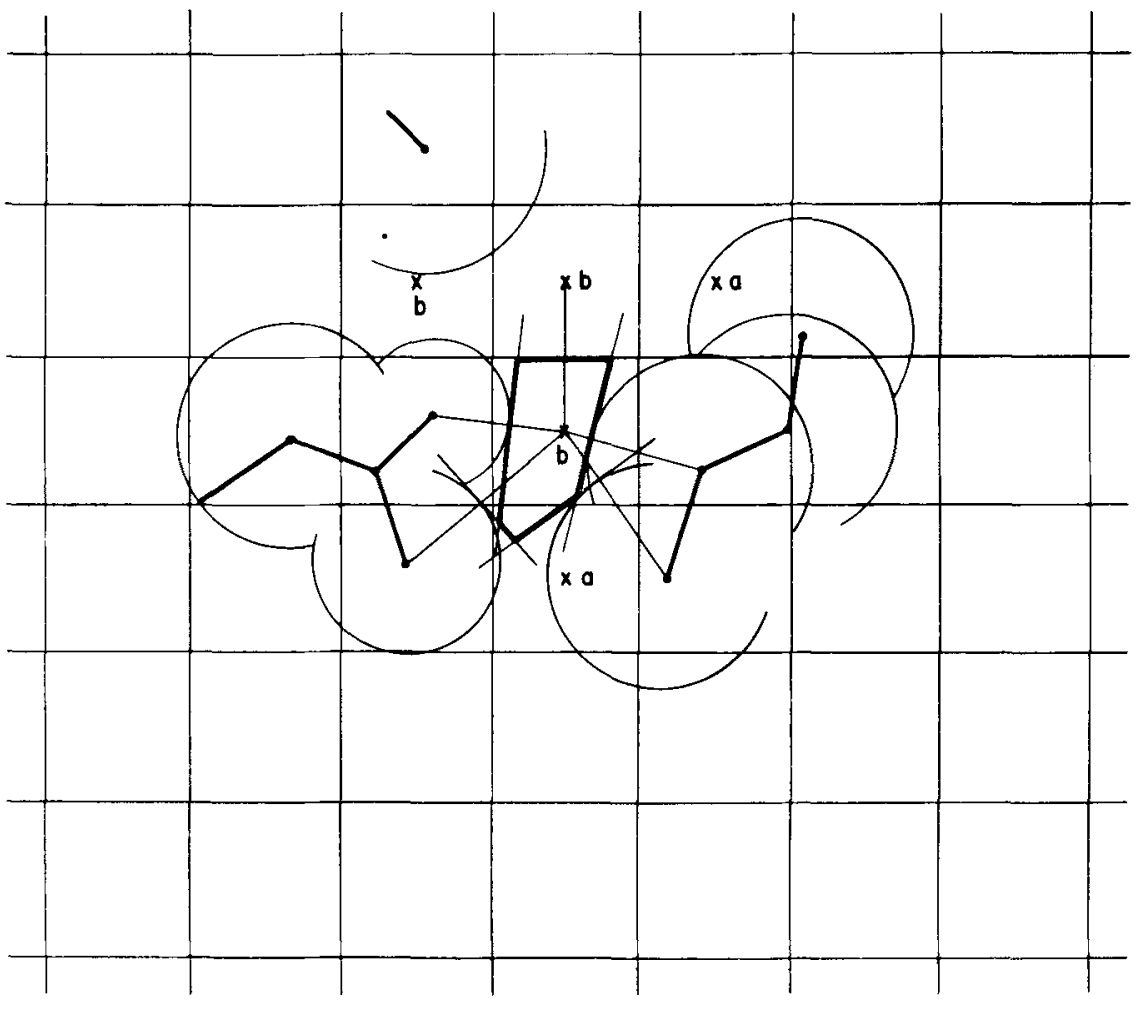

Carlsberg Res. Common. Vol. 44, p. 47-63, 1979

51 
isothermal compressibility. From estimates of this parameter it is possible to calculate fluctuations in packing defect volumes based on actual $\mathrm{X}$-ray structures.

\section{AN ELEMENTARY THEORY OF FLUCTUATIONS IN PROTEIN STRUCTURE}

\subsection{The Voronoi procedure}

The Voronoi construction on any set of points in three dimensions produces an equivalent set of irregular, connected polyhedra, one polyhedron surrounding each point (see, for example, CoXETER, (9)). Each polyhedral face is part of a plane which bisects the vector between a pair of points. The volume of each polyhedron is uniquely defined by the geometry of the points. The collection of polyhedra account exactly for all of the space occupied by the points. If the set of points is finite, the polyhedra for most points at the surface of the set are undefined (Figure la). Thus for calculation on the full set of atomic positions of a protein, for example, it is necessary to generate a hypothetical surface layer to enclose

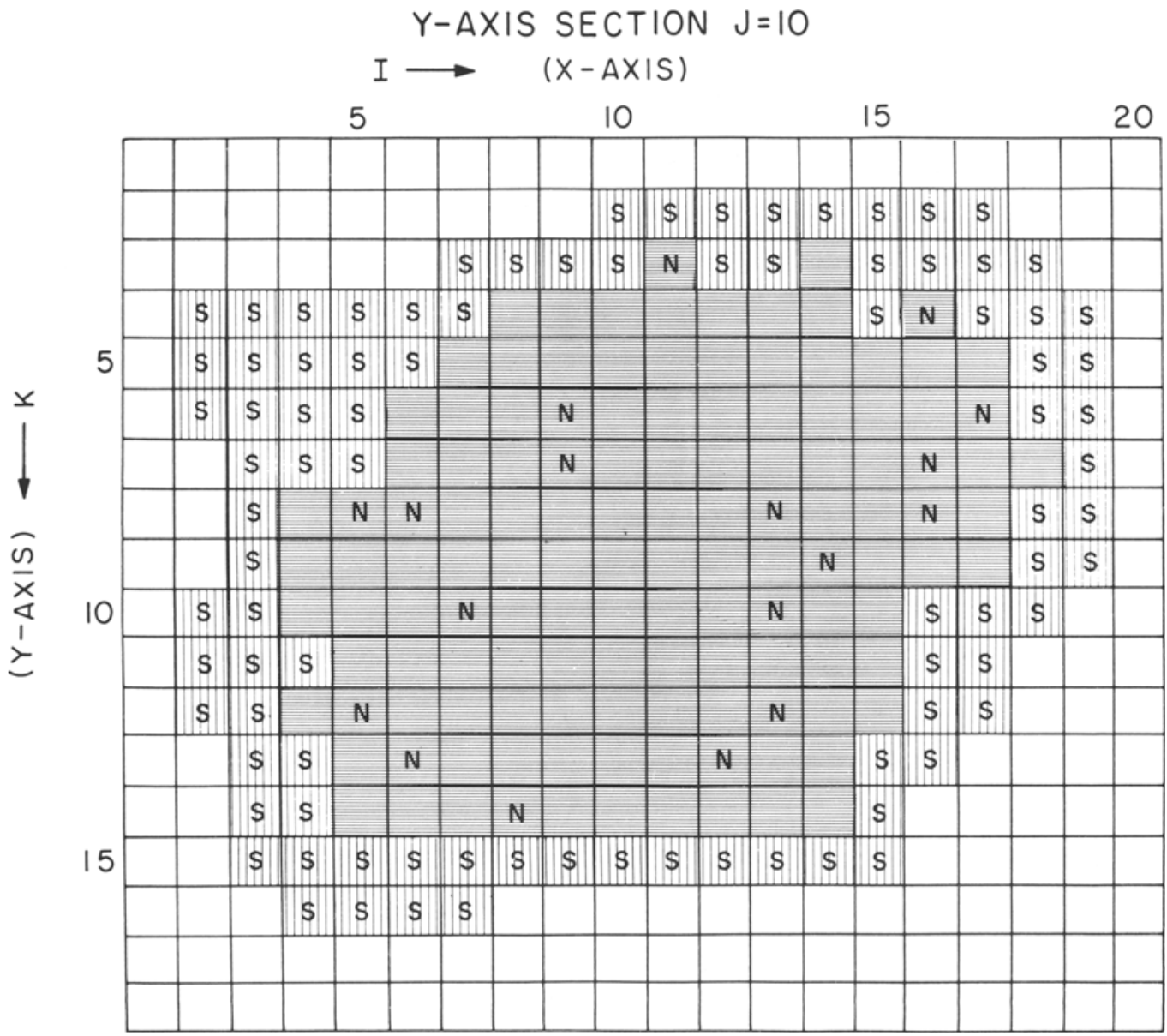

Figure 2. One $\mathrm{x}, \mathrm{z}$ section through the cubic lattice for myoglobin. The edge of the unit cube is $2.8 \AA$. The numbers are the $i, j$ or $k$ indices identifying the cube locations. The horizontally shaded cubes are those assigned to protein atoms. The symbol $\mathrm{N}$ identifies those cubes containing a main chain peptide nitrogen atom. The vertically shaded cubes labeled $S$ are those assigned as the surface, or SHELL, layer surrounding the protein. Those not in contact with protein atoms in this section are part of the SHELL structure of contact with protein containing cubes in the layers above or below the area shown. 
Table I

Description of lattice positions for myoglobin

1. Protein atom distribution:

Number of cells, $n$, with i protein atoms: $n / i=366 / 1 ; 189 / 2 ; 113 / 3 ; 38 / 4 ; 5 / 5$.

Total number of protein atoms

$=1260$

2. Number of cells containing 1 or more protein atoms

$=711$

3. Number of cells with centers inside the protein van der Waals envelope

$=108$

Total number of cells assigned to protein atoms $(2 .+3$.

$=819$

Total number of cells in SHELL envelope

$=1087$

Number of empty cells (cavities) with centers outside the protein van der Waals envelope

$=290$

Total number of cells inside SHELL envelope $(4 .+6$.)

$=1109$

the protein atoms. One method for doing this is to set up a cubic lattice into which the protein is inserted (see ref. 29, 30, 33). Lattice points next to the protein atoms are then used to define this hypothetical surface layer (Figure 2). (The nature of the layer chosen will affect the calculated volumes of the neighboring atoms. This problem has been discussed by Richards (33) and FINNEY (14) but is not of concern in this paper.)

\subsection{Description of the cubic lattice and the surface envelope}

The lattice was assigned a repeat distance of $2.8 \AA$. This value was originally chosen to be equivalent to the oxygen-oxygen distance in ice (33). A lattice cube will thus just enclose a hypothetical water molecule represented by a sphere of diameter $2.8 \AA$. The lattice was oriented to match the Cartesian coordinates for the protein atoms. The protein coordinates were converted to indices and all cells which contained the center of one or more protein atoms were flagged.

The hypothetical solvent layer around the protein positions was called SHELL (Figure 2) and was developed as follows. All cells not containing protein atoms were checked, and those for which at least one of the 26 surrounding lattice positions was occupied by a protein atom(s) were flagged as potential members of SHELL. This first list contained all of the final positions but also a number of vacant cells inside the protein which were clearly inappropriate as part of an external envelope. The latter were eliminated by checking diametrically opposite pairs among the 26 neighbors. If both members of any pair contained protein atoms, the central cell was considered too tightly packed to serve as part of the solvent envelope, was specially flagged, and was eliminated from the SHELL list.

In the original study (33), the empty cells, which were identified as being inside the final SHELL positions, were assigned to the protein. The volume of space that they represented appeared in the Voronoi polyhedra calculated for the protein atoms. Although such cells did not contain a protein atom center, they did frequently have the van der Waals envelopes of atoms in neighboring cubes running through them. It was thus perfectly reasonable to divide this space up among the protein atoms. The observed spread in packing density through the protein reflected the inclusion of this space. In the present work these interior, but empty, cells have been looked at more closely and considered as packing defects. A summary of the present cell assignments for myoglobin is given in Table I.

\subsubsection{Specification of packing defects}

The empty cells inside SHELL fall into two categories. For the first the cell centers are actually inside the van der Waals envelope of some protein atom. Nothing useful can be done with these and they are simply assigned to the protein. In the second category, the center is outside the van der Waals envelope of all protein atoms. Such centers can be used as points in the Voronoi construction along with the protein atoms.

In an attempt to realistically estimate the defect volume, for vectors connecting the defect 


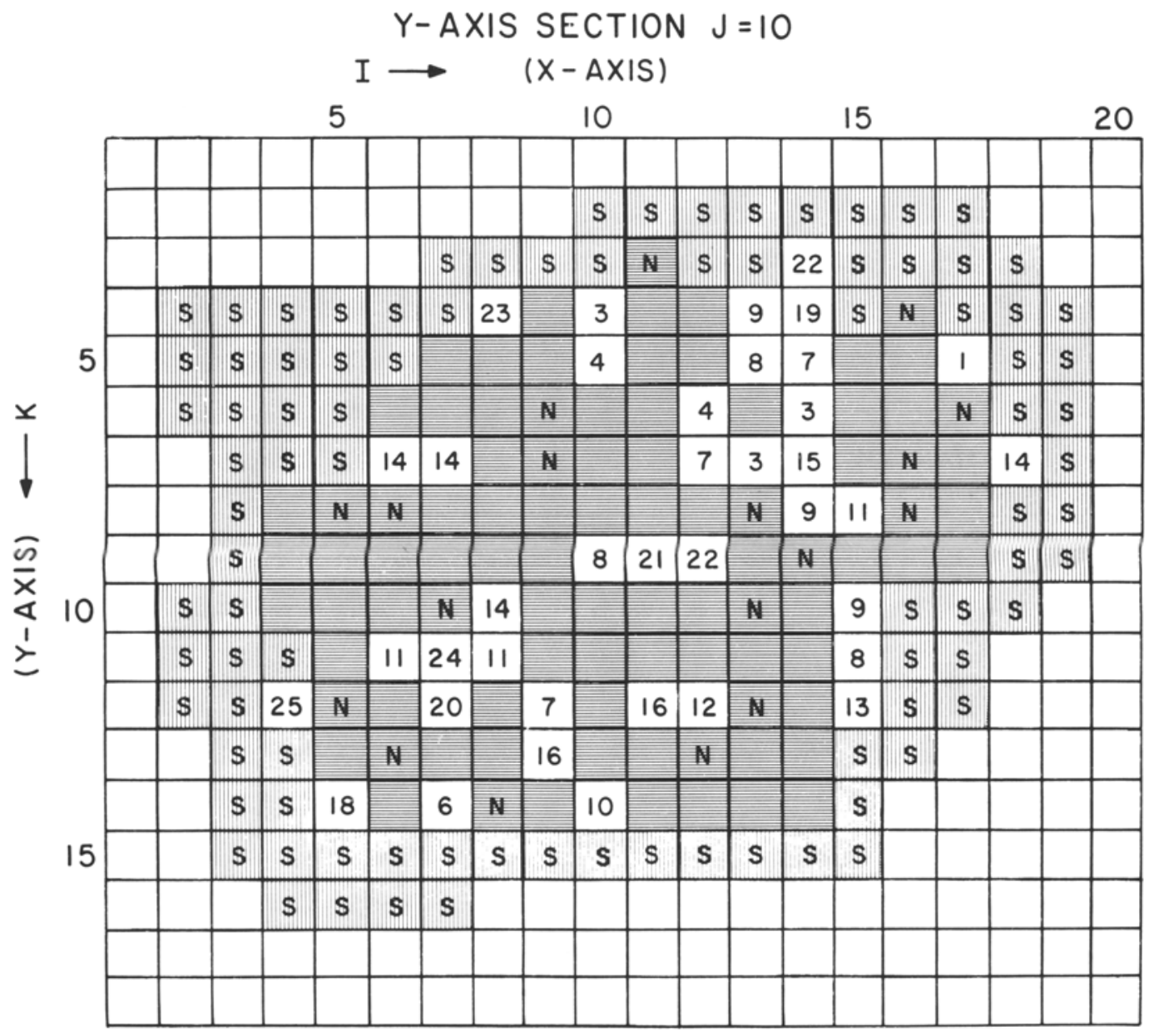

Figure 3. Packing defects in a section of the myoglobin lattice. The same section as Figure 2 is shown. The symbol keys are given in the legend of that figure but in addition the clear cubes represent the defects. The numbers are the Voroni defect volumes in $\AA^{3}$.

cells with protein atoms were not bisected. Instead, the perpendicular planes were drawn tangent to the van der Waals envelope of the protein atoms (Figure lb). All other vectors, protein-protein and empty cell-empty cell, were bisected as usual. This removed the geometrical perfection of the construction and some volume error was introduced, but it is unlikely to be a serious problem for present purposes (see Richards, (33) for further discussion).

The result of a calculation including these defect positions is a reduction in the volume assigned to the protein atoms. The mean residue volumes reported by СнотніА (5) decrease 13-
$15 \%$ but the standard deviations are almost halved since much of the variable empty space originally included is now being counted separately. Each empty cell has a calculated volume; the variance is large but the mean for myoglobin is about $12 \AA^{3}$. These defects are scattered throughout the structure, but about one-half are at the surface reflecting the uncertainties in establishing the original SHELL envelope. The location and volume of the defects in a section of the lattice are shown in Figure 3. Many of the defects are connected in patches which proceed from the surface for varying distances toward the interior of the protein. 
There is no simple way of describing an irregular cavity. There is no set of atom centers to serve as starting points. The cubic lattice approach is one possible approximation. The centers of contiguous empty cells serve as a framework for describing a cavity. These cells have no physical significance but can be used in calculations of both volume and shape. The packing defects are quantitatively described by their lattice positions and the Voronoi volumes (convex polyhedra) assigned to them. The next level of description provides some measure of the connectivity of these positions. For each defect the 26 neighbors are examined for additional vacant cells which may be part of a larger defect structure (Figure 4). There are 6 face connected positions $(F)$ which are the true nearest neighbors only the lattice spacing, $d$, away. There are

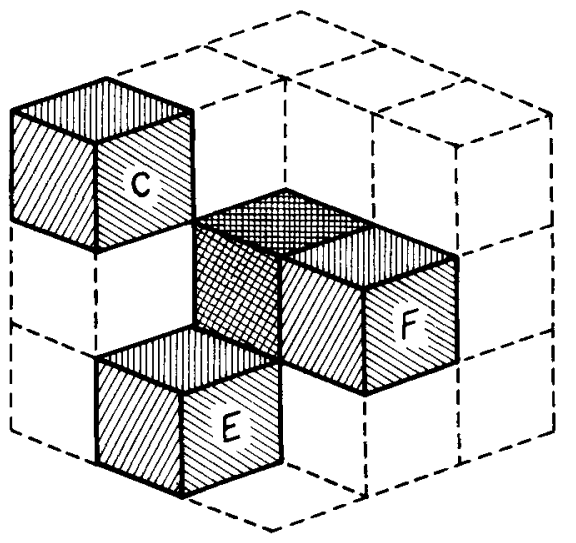

Figure 4. Section of cubic lattice showing positions of face, F, edge, E, and corner, C, connected neighbors of a particular central cube.

CELL CONNECTION MODE

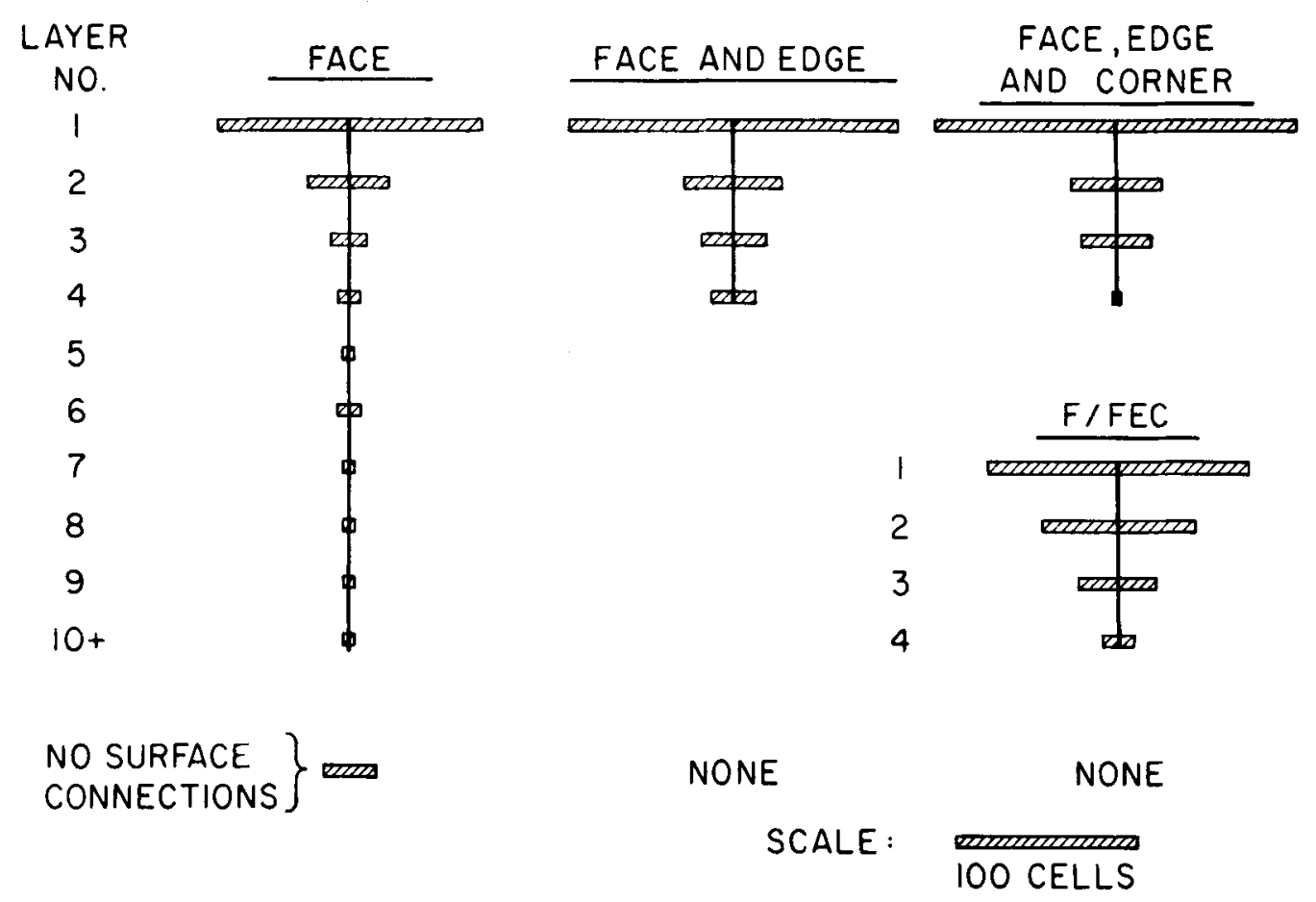

Figure 5. Nature of the connectivity of the packing defects for different modes of connection. In layer 1 the defects are in direct contact with the SHELL surface locations with at least one connection of the mode type indicated. Layer 2 is in contact with the layer 1 positions but not with SHELL positions etc. The numbers of cubes in each layer is shown by the length of the bars. The total number of defects for the particular lattice is 290. 
Table II

Volumes of defect cells in myoglobin

\begin{tabular}{|c|c|c|c|c|}
\hline $\begin{array}{l}\text { Locationa } \\
\text { Volume }-\AA^{3} \\
\text { Number of cells }\end{array}$ & & Conned & lodeb & \\
\hline Mean volume per cell & $\mathrm{F}$ & FE & FEC & F/FEC \\
\hline External & & & & \\
\hline $\mathrm{V}$ & 2267 & 2628 & 2794 & 2267 \\
\hline $\mathrm{n}$ & 145 & 179 & 198 & 145 \\
\hline $\mathrm{v}$ & 15.6 & 14.7 & 14.1 & 15.6 \\
\hline Internal with connection to surfac & & & & \\
\hline V & 1169 & 1019 & 853 & 1380 \\
\hline $\mathrm{n}$ & 118 & 111 & 92 & 145 \\
\hline $\mathrm{v}$ & 9.9 & 9.2 & 9.3 & 9.5 \\
\hline Internal with no connection to surf & & & & \\
\hline $\mathrm{V}$ & 211 & 0 & 0 & 0 \\
\hline $\mathrm{n}$ & 27 & 0 & 0 & 0 \\
\hline $\mathrm{v}$ & 7.8 & - & - & - \\
\hline Total volumes $-\AA^{3}$ & & & Latti & ellsc \\
\hline Protein - all space inside SHELL & & & & \\
\hline - all cavities omitted & & & & \\
\hline Cavities & & & & \\
\hline
\end{tabular}

a Cells with one or more neighbors from SHELL are called external. All others are internal. Internal cells with no neighbor connections to SHELL are separately noted.

$b$ See text for description.

c Volumes in this column are obtained by multiplying the appropriate number of cells by $21.95 \AA^{3}$, the volume of a single cell.

twelve edge connected neighbors $(E)$ at a distance of $\sqrt{2} \mathrm{~d}$ and eight corner connected positions (C) at a distance of $\sqrt{3} \mathrm{~d}$. Connectivity of the defect cells can then be traced at different levels: considering only face connection, $F$, face and edge, FE, or all possible neighbors, FEC. For a given set of defect cubes, the connectivity assigned will depend on the connection mode chosen (Figure 5). Some volumes and averages are shown in Table II.

\subsubsection{Volume fluctuations}

For a system of total volume, $\mathrm{V}$, the mean square volume fluctuation, $\overline{\delta \mathrm{V}^{2}}$, is related to the isothermal bulk compressibility, $\beta_{\mathrm{T}}(8,2)$ :

$$
\overline{\delta V^{2}}=k T V \beta_{T}
$$

If the system is considered to be a protein molecule such a volume fluctuation will be spread over the whole molecule. Since the actual protein atoms are essentially incompressible in the energy range of interest here, the expansion and contraction of cavities is the only way to generate this volume fluctuation. If the number of defect cells is $n$, and the mean volume per cell is $\mathrm{v}$, then for equivalent and independent fluctuations in each cell:

$$
\overline{\delta V^{2}}=n \overline{\delta v^{2}}
$$

The root mean square volume fluctuation for the defect cell $\delta v_{\text {rms }}$ may be used as the standard deviation, $\sigma$, for a gaussian distribution. The probability, $\mathrm{P}$, that a given defect, $\mathrm{i}$, will have a volume equal or greater than $v_{0}$ can be calculated 


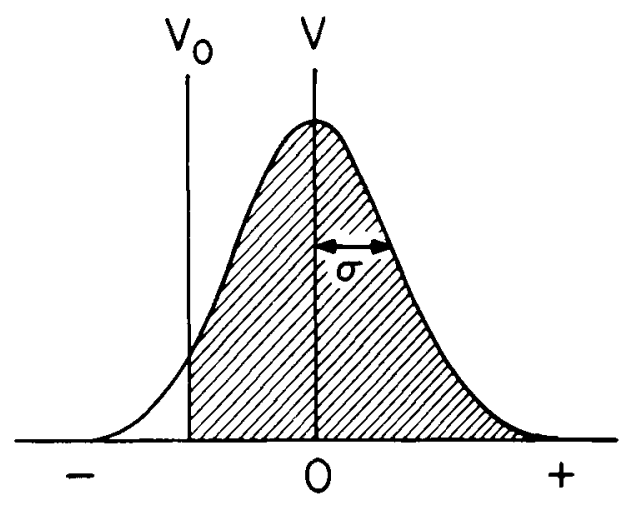

$x$

$$
x=\frac{V_{0}-V}{\sigma \sqrt{2}}
$$

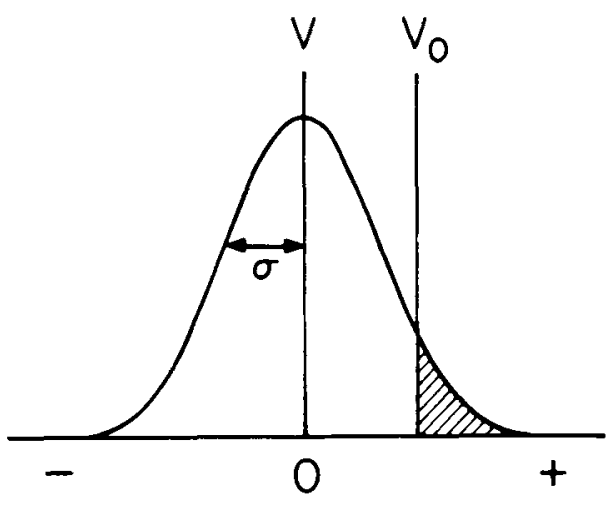

$x$

$$
\begin{gathered}
V>V_{0} \\
P=1 / 2(1+\operatorname{erf} x) \\
\text { 6. Normal distributions. The shaded areas } \\
\text { the standard error function, Figure 6. } \\
\begin{array}{c}
P_{i}\left(v_{0}\right)=\frac{1}{2}(1-\operatorname{erf}(x)) \quad v_{i} \leq v_{0} \\
=\frac{1}{2}(1+\operatorname{erf}(x)) \quad v_{i} \geq v_{0}
\end{array}
\end{gathered}
$$

$P=1 / 2(1-\operatorname{erf} x)$

Figure 6. Normal distributions. The shaded areas represent the probability, $P$, of $v \geq v_{0}$.

from the standard error function, Figure 6. If $v_{i}$ is the Voronoi volume of the defect cell $i$, then:

where $x=\frac{v_{0}-v_{i}}{\sqrt{2} \sigma}$. The volume, $v_{0}$, is an adjustable parameter. If one is considering the entry of a solvent or solute molecule into the protein, $v_{0}$ is set at an assumed value appropriate for the size of this entering molecule. In this scheme such a probe would follow a channel to the protein interior which develops through fluctuations in linked cavity volumes.

\subsubsection{Channels to the interior}

For access to the interior through channels of cavities the individual defect probabilities must be combined, making use of the known connectivity of the defect cells. The probability, $Q_{j}$, that the cell, $\mathrm{j}$, will contain a probe molecule, is given by

$$
Q_{j}=\left[1-\prod_{i=1}^{26}\left(1-\frac{m_{i}}{l_{i}} Q_{i}\right)\right] P_{i}
$$

with the product taken over all 26 neighboring cells. $P_{j}$ is calculated from equation [3]. The factor $m_{i}$ is a mode or identity index. If the neighbor cell $\mathrm{i}$ is assigned to protein atoms, $\mathrm{m}_{\mathrm{i}}=0$. If $\mathrm{i}$ represents another defect cell or a SHELL position, then $m_{i}$ is set equal to 0 or 1 depending on the connection mode chosen for the calculation $(F, F E$, etc.) and the lattice position ( $F, E$ or $C$ ) of $i$ with respect to $j$. The factor $L_{i}$ is related to the diffusion path length from $i$ to $j$. If the cells are $F$ connected $l=1$, for E connection $\mathrm{l}=2$, for $\mathrm{C}$ connection $\mathrm{l}=3$. These values represent the squares of the ratios of the actual distances to the length of the cell edge. The assumption is made that the time for diffusion of the probe from one cell to the next occurs in a time interval comparable to or slower than the volume fluctuation frequency. In free diffusion a water molecule will move a distance of $2.8 \AA$ in about $10^{-11} \mathrm{sec}$. Low frequency motions of the type principally responsible for the fluctuations of interest here are probably in the neighborhood of $10 \mathrm{~cm}^{-1}$ (31) or higher corresponding to a period of $10^{-11} \mathrm{sec}$ or less. If diffusion limitation is not involved, then the factor $l_{i}$ is set equal to 1 . Using equations [3] and [4] the probability that each defect cell is occupied can be calculated starting with those adjacent to SHELL positions and then working down the connectivity chain to the interior positions. 


\subsection{Parameter selection}

Each cell in the cubic lattice has a volume of $2.8^{3} \AA^{3}$ or $21.95 \AA^{3}$. This is too small for a solvent molecule in bulk water where the mean volume is about $30 \AA^{3}$. The actual packing volume of water at the protein surface is not known but on the average is likely to be close to that of bulk water. The probability that a SHELL position in the lattice is occupied is taken as the volume ratio $21.95 / 30$ or about 0.7 . On occasion this might be considered an adjustable parameter as discussed below.The value of the isothermal compressibility, $\beta_{\mathrm{T}}$, for proteins is not known precisely but may be in the range of $10^{-11}$ to $10^{-12} \mathrm{~cm}^{2} \mathrm{dyne}^{-1}$. If the system is a myoglobin molecule with a volume of 21,500 $\AA^{3}$, the probable range for $\left(\overline{\delta V^{2}}\right)^{\frac{1}{2}}$ would be 30 to $95 \AA^{3}$ (see COOPER, (8)). In the present example, the number of defect cells is 290 . Thus from equation [2], the range for the volume fluctuations of individual cells, $\delta \mathrm{v}_{\mathrm{rms}}$, would be 1.8 to $5.5 \AA^{3}$. A trial value of 4.0 has been used as a starting estimate for $\sigma$ in equation [3]. If the interior were liquid-like, $\beta_{\Upsilon}$ might approach $10^{-10} \mathrm{~cm}^{2}$ dyne ${ }^{-1}$ corresponding to a $\delta v_{\mathrm{rms}}$ of about $17 \AA^{3}$. (A recent measurement by MiLLERO et al. (26) for lysozyme sets $\beta_{\mathrm{T}}=45 \times$ $10^{-12} \mathrm{~cm}^{2} \cdot$ dyne $^{-1}$.)

For a process such as hydrogen exchange the entering probe can be considered a water molecule or closely related ionic species. The nature of the true diffusing species for this process is uncertain and its volume characteristics when isolated in the interior of the protein certainly unknown. The factor $v_{0}$ has been assigned a starting value of $30 \AA^{3}$, but must be regarded as an adjustable parameter.

\subsection{Hydrogen exchange}

Hydrogen exchange of peptide protons is both an acid and base catalyzed reaction, the rate of exchange with neutral water molecules is reported to be small (see ref. 13, 18, 19, 41). The intrinsic rate of a specific proton will depend on the flanking amino acid residues, the primary structure. In a large peptide, the rate will be influenced by the secondary structure of which the particular amide is a part. In a protein the further protection of the amide by the tertiary structure will be an additional factor. The rate equation for the single main chain proton in residue $i$ may thus be written as

$$
H_{i}^{\text {rem }}=\exp \left(-c_{i} b_{i} a_{i} k_{o}\right) t
$$

where $\mathrm{H}_{\mathrm{i}}^{\mathrm{rem}}$ is the fraction of the amide proton label on residue $i$ remaining at time $t, k_{0}$ is the exchange rate for polyalanine under the particular conditions of the experiment, and the other factors represent the influence of the primary, $a_{i}$, secondary, $b_{i}$, and tertiary, $c_{i}$, structure of the protein. Values for estimating $a_{i}$ are provided by Molday et al. (27) and may be greater than or less than 1 . The factors $b_{i}$ and $c_{i}$, always less than unity, are not easily separated and are combined in this study as $\lambda_{\mathrm{i}}=\mathrm{b}_{\mathrm{i}} \mathrm{c}_{\mathrm{i}}$. The actual motions by which the volume fluctuations occur are not specified but are assumed to provide not only the change in tertiary structure giving solvent access to the interior but also sufficient distortion in secondary structure to permit proton exchange to occur. For many test calculations $a_{i}$ has been assumed equal to 1 . Then for a protein with $n$ exchangeable amide protons:

$$
H_{\text {totat }}^{\text {rem }}=\sum_{i=1}^{n} e^{-\lambda_{i} k_{o} t}
$$

The factor $\lambda_{i}$ may be thought of as the probability that the amide proton is available for exchange. This formalism permits a large amount of data covering a huge time range to be codified and does not require specification of the mechanism by which the protons become accessible for reaction (18). In this study we assume that the solvent is brought to the amide through fluctuations in cavity volumes, and that these fluctuations permit calculation of the $\lambda_{i}$ values.

The lattice position containing a main chain amide nitrogen atom is surveyed for surrounding cavity cells. The probability, $Q_{j}$, that each of the latter is occupied by a solvent molecule has already been calculated as described above. At this stage no detailed exchange mechanism is postulated. The assumption is made that appearance of a solvent molecule in a neighboring cell will result in exchange with a fixed probability independent of any local aspect of the protein structure.

$$
\lambda_{i}=1-\prod_{j=1}^{26}\left(1-\frac{m_{j}}{l_{j}} Q_{j}\right)
$$


$\mathrm{Q}, \mathrm{m}$ and 1 all have the same definitions given for equation [4]. Since a given amide position may have more than one cavity cell as a neighbor, there may be several paths by which a solvent molecule may approach the exchangeable proton. The predicted exchange behavior of a given amide is thus intimately tied to the protein structure.

\subsection{Calculated exchange curves}

Equations 3, 4, 6, and 7 have been used to calculate $\mathrm{H}^{\mathrm{rem}}$ v.s. $\log \left(\mathrm{k}_{0} \mathrm{t}\right)$ for various values of the adjustable parameters. In Figure 7 it can be seen that the shape of the curves depends dramatically on the connection mode chosen for the calculation. In the $\mathrm{F}$ mode a large number of amide protons are predicted to be totally unexchangeable, not in agreement with experiment. In the FE or FEC modes all protons are eventually exchangeable at long times but many of the groups are so close to the surface that the number of rapidly exchanging protons is very high. The general trend of the actual data are

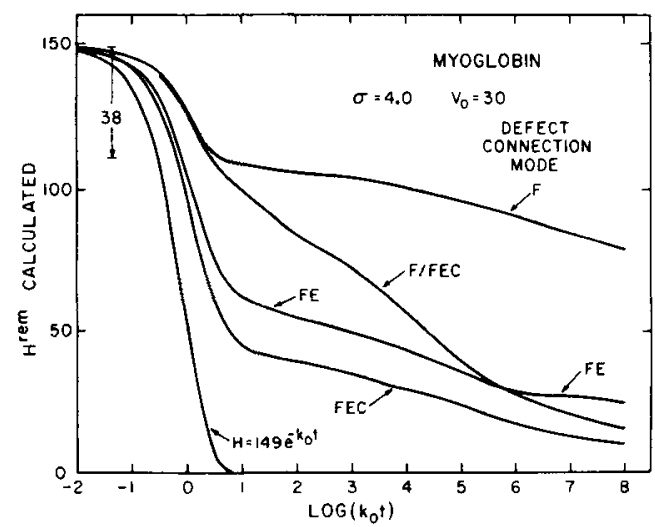

Figure 7. Hydrogen exchange curves based on the myoglobin X-ray coordinate data. The lines were computed using equations $3,4,6$ and 7 with the parameters $\sigma=4.0, v_{o}=30$ and the connection mode indicated. If all the protons exchange at a single rate $\mathrm{k}_{\mathrm{o}}$ unmodulated by the protein structure, then the data would fall on the single exponential decay shown as the left hand curve. Of the amide $\mathrm{N}$ atoms in the cubic lattice, 38 have direct access to SHELL positions. At the present level of approximation these will all exchange at the maximum rate $k_{0}$ independent of the defect structure.

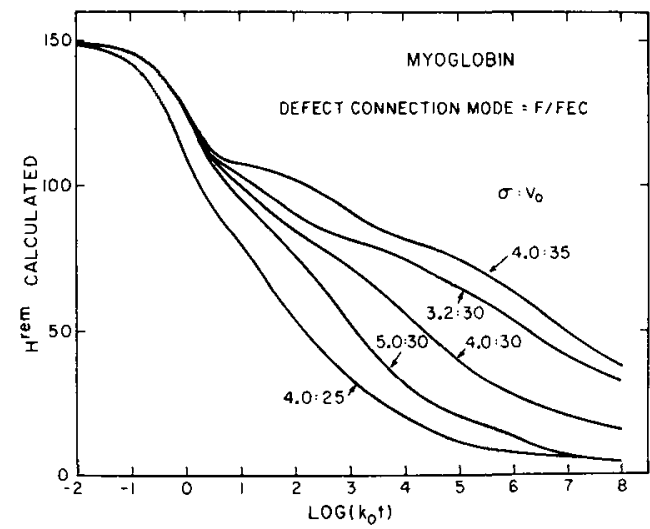

Figure 8. Calculated hydrogen exchange curves for myoglobin with the mixed connection mode F/FEC. The influence of the parameters $\sigma$ and $v_{0}$ on curve shape and position is shown.

better fit by the mixed mode curve F/FEC. In this procedure the probe molecules enter the protein at the first cavity layer, or directly at surface amide protons, only through $\mathrm{F}$ connected cubes. Once inside they proceed through the interior regions using the full $\mathrm{FEC}$ connection mode. The general curve shape is closer to those seen for many proteins in general and for myoglobin in particular.

The sensitivity of the calculated curves to the parameters $v_{0}$ and $\sigma$ is shown in Figure 8. Quite small changes in these parameters have a large

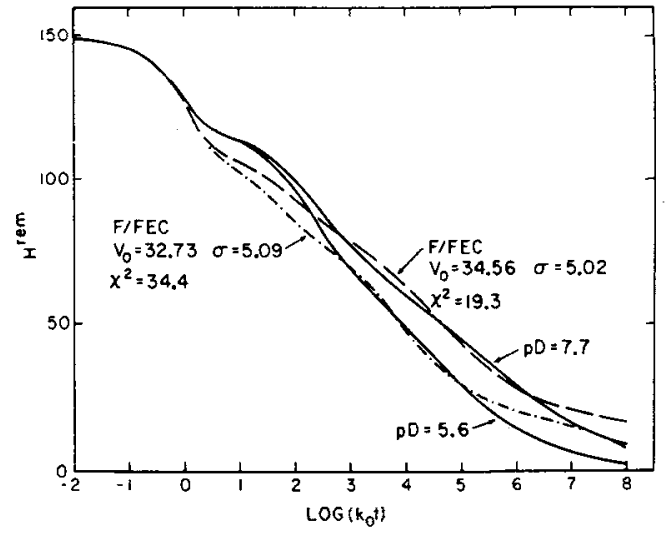

Figure 9. Calculated hydrogen exchange curves for myoglobin. Attempts to fit the experimental data at pH 5.6 and 7.7 (28) using the F/FEC mode with least squares variation of $\sigma$ and $v_{0}$. The chi square estimates of errors are shown. 
effect on the more slowly exchanging groups remembering that the time scale shown is logarithmic.

The data on hydrogen exchange in sperm whale myoglobin as reported by NAKANISHI et al. (28) are shown in Figure 9. Superimposed are the first attempts to fit these curves by standard least squares procedures varying both $\sigma$ and $v_{0}$. The protein coordinate data reported by TAKANO (40) were obtained from the Protein Data Bank at the Brookhaven National Laboratory, File $3 \mathrm{MBN}$ (1). The curve below zero on the time scale is not actual data but simply an extrapolation and tends to bias the least squares fit. The parameters $\sigma$ and $\mathrm{v}_{\mathrm{o}}$ interact strongly and lead to very slow convergence of the least squares program. The fit is clearly poorest in the early time region around $\log \left(\mathrm{k}_{\mathrm{o}} \mathrm{t}\right)=1$.

An additional restraint on the initial access of solvent through the interface was imposed by reducing the SHELL site occupancy from 0.7 to 0.3 . There is no physical reason for this at the moment; the occupancy is just used as a convenient adjustable parameter. The time range has been restricted to 0 to 8 on $\log \left(k_{0} t\right)$, and $v_{0}$ and $\sigma$ were refined on alternating least squares cycles. The result for myoglobin is shown in Figure 10.

The exchange process depends on the properties of water and on the detailed mechanism at the amide nitrogen atom. The parameter $v_{0}$ is

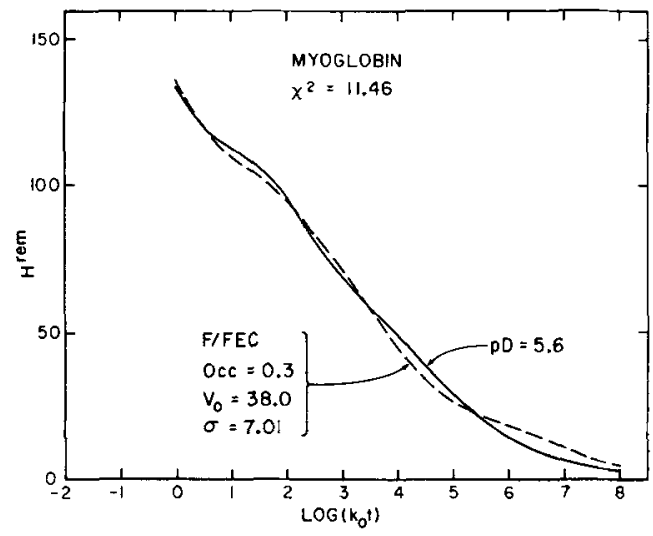

Figure 10. Calculated hydrogen exchange curve for myoglobin. Attempt to fit the pH 5.6 data in the $\log$ $\left(k_{0} t\right)$ range of $0-8$ with a lowered surface accessibility parameter (see text). $v_{0}$ and $\sigma$ were varied individually in alternate least squares cycles.

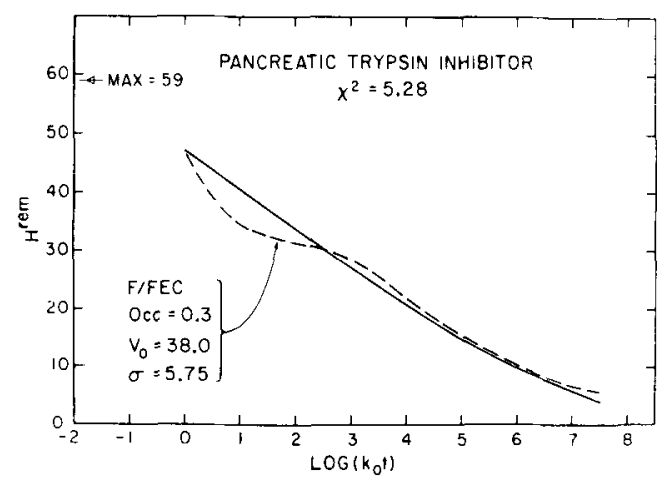

Figure 11. Calculated hydrogen exchange curve for the basic pancreatic trypsin inhibitor with $\sigma$ as the only parameter adjusted. Other parameters the same as Figure 10. The data (solid line) are from HvidT and PedesRsen (20).

related to this process and should be independent of the protein whose exchange is being considered. The parameter $\sigma$ on the other hand is a property of the protein. The fit to the data of HVIDT and PEDERSEN (20) on bovine pancreatic trypsin inhibitor allowing $\sigma$ as the only adjustable parameter is shown in Figure 11. The lower value of $\sigma$ is in agreement with the general impression that this small protein is 'tight'. (See also ref. 42.)

\section{DISCUSSION}

The hydrogen exchange data for many proteins reflect a range of at least eight orders of magnitude in the rate constants for individual amide protons. On the basis of the calculations described above, thermally induced fluctuations in the size of the packing defects present in all proteins appear sufficient to account for this distribution of exchange rates as suggested by LUMRY and ROSENBERG (25). Large structural changes involving movements of more than 1 to $2 \AA$ are not required. (This study is not concerned with the question of whether or not large structural changes actually occur, it simply shows that they are not necessary.)

Given that all proteins have roughly the same composition, the apparent volume, $v_{0}$, required to accommodate a water molecule should be independent of the particular protein in question. 
The parameter $\sigma$, however, is clearly a property of the macromolecule and would be expected to vary for different proteins and for the same protein under different conditions. With myoglobin and for the two trypsin inhibitors the numerical values of the parameters are within physically reasonable ranges, but no detailed interpretation is warranted at this time.

A given protein under defined conditions will be characterized by a particular value of $\sigma$ no matter what technique is used to examine the fluctuations. The $\mathrm{O}_{2}$ molecule is not very different in size from a water molecule and thus the 'permeability' of the protein to the two probes would be expected to be comparable. Yet the contact quenching by $\mathrm{O}_{2}$ of the fluorescence of tryptophan residues in a number of proteins indicates that $\mathrm{O}_{2}$ access to the protein interior is reduced by less than a factor of 10 below the free diffusion estimate (21). This apparent discrepancy with hydrogen exchange estimates may be simply a target size problem. The indole ring of tryptophan is quite large and there will be many defect paths to its surface. In most instances at least one of these paths will have a high probability. Calculations for $\operatorname{Trp}$ and Tyr residues in myoglobin show this to be the case. Effective quenching will thus occur even if most of the ring is tightly packed and not available to the oxygen. In contrast the amide proton is a small target and may easily have a low probability path leading to it with the resultant dramatically reduced exchange rate.

The theory predicts a very steep dependence of access probability on $\mathrm{v}_{\mathbf{0}}$. Thus the protein rapidly becomes impenetrable as the probe increases in size. The decrease in fluorescence quenching efficiency as the quencher becomes much larger than oxygen appears to bear this out.

In the calculations an estimate is made of the accessibility of each amide residue separately. In principle the individual exchange rates can be rank ordered. At this point the theory fails because of the gross approximations involved. The assumptions made for the volume fluctuations of each defect are: a) the structural alterations are isotropic; b) separate defects fluctuate independently; c) the fluctuations can be described by a normal curve; d) the parameters describing the fluctuations are constant and independent of the position of the defect in the protein. In addition sequence variation of intrinsic exchange rate has not been taken into account. All of these assumptions are probably incorrect. Due to some cancellation of errors, the overall behavior of the system may be qualitatively correct, but the details will surely be wrong.

The most obvious evidence of errors is in the fast exchange region, those amides which are in direct contact with the solvent SHELL. It is here that the failure to directly and specially account for primary and secondary structure effects on exchange rates will be most obvious. The interior amides will frequently require sufficient defect expansion to move neighboring atoms more than $0.5 \AA$. This would be enough to break a hydrogen bond and thus remove the secondary structure restriction at the same time that the cavity is being produced to contain the solvent molecule. This may often not be required at the surface and thus the secondary structure effect will not be accounted for. The primary structure would affect all amide protons regardless of location if the chemical exchange mechanism remains the same.

The motions that the atoms undergo are likely to be anharmonic and certainly anisotropic. They will also be coupled for the low frequency events of particular concern here. Proper evaluation of realistic motional modes will require further study and more sophisticated approaches.

\section{REFERENCES}

1. Bernstein, F. C., T. F. Koetzle, G. B. J. Williams, E. F. Meyer, M. D. Brice, J. R. Rodgers, O. Kennard, T. Shimanouchl \& M.TASUMI: The protein data bank: A computerbased archival file for macromolecular structures. J. Mol. Biol. 112, 535-542 (1977)

2. CAllen, H. B.: Thermodynamics. John Wiley and Sons, New York, pp. 267-283 (1960)

3. CARERI, G.: The fluctuating enzyme. In: Quantum statistical mechanics in the natural sciences. B. Karsunoglu, S. L. Mintz \& S. M. Widmayer, eds., Plenum press, N. Y., pp. 15-35 (1974)

4. Снотнia, C.: Hydrophobic bonding and accessible surface area in proteins. Nature 248, 338339 (1974)

5. Снотніа, C.: Structural invariants in protein folding. Nature 254, 304-308 (1975) 
6. Chou, P. Y. \& G. D. Fasman: Prediction of the secondary structure of proteins from their amino acid sequence. Adv. Enzymology 47, 45-148 (1977)

7. Cohen, F.: Protein Folding. B. S. Dissertation, scholar of the house program, Yale University (1978)

8. CoOper, A.: Thermodynamic fluctuations in protein molecules. Proc. Natl. Acad. Sci. USA 73, 2740-2741 (1976)

9. Coxeter, H. S. M.: Introduction to geometry. John Wiley \& Sons, N. Y. (1961)

10. Creighton, T. E.: Experimental studies of protein folding and unfolding. Prog. Biophys. Molec. Biol. 33, 231-297 (1978)

11. Eftink, M. R. \& C. A. Ghiron: Dynamics of a protein matrix revealed by fluorescence quenching. Proc. Natl. Acad. Sci. USA 72, 32903294 (1975)

12. Eftink, M. R. \& C. A. Ghiron: Exposure of tryptophanyl residues in proteins. Quantitative determination by fluorescence quenching studies. Biochemistry 15, 672-680 (1976)

13. Englander, S. W., N. W. Downer \& H. Teitelbaum: Hydrogen Exchange. Ann. Rev. Biochem. 41, 903-924 (1972)

14. FinNeY, J. L.: Volume occupation, environment and accessibility in proteins. The problem of the protein surface. J. Mol. Biol. 96, 721-732 (1975)

15. FinNey, J. L.: Volume occupation, environment and accessibility in proteins. Environment and molecular area of RNase-S. J. Mol. Biol. 119, 415-441 (1978)

16. Gurd, F. R. N. \& M. Rothgeb: Motions in proteins. Adv. Prot. Chem. 33, (1979) (in press)

17. HermanN, R. B.: Theory of hydrophobic bonding. II. The correlation of hydrocarbon solubility in water with solvent cavity surface area. J. Phys. Chem. 76, 2754-2759 (1972)

18. HvidT, A.: Isotopic hydrogen exchange in solutions of biological macromolecules. In: Dynamic aspects of conformation changes in biological macromolecules, C. Sadron, ed., D. Reidel Publ. Co., Dordrecht, Holland, pp. 103115 (1973)

19. HvidT, A. \& S. O. NiELSEN: Hydrogen exchange in proteins. Adv. Prot. Chem. 21, 287-386 (1966)

20. Hvidt, A. \& E. J. Pedersen: A Comparative study on the basic pancreatic trypsin inhibitor and insulin by the hydrogen exchange method. Eur. J. Biochem, 48, 333-338 (1974)

21. LAKowicz, J. \& G. WeBER: Quenching of Fluorescence by oxygen. A probe for structural flucations in macromolecules. Biochemistry 12 , 4161-4170 (1973)

22. LEE, B. \& F. M. RichaRds: The interpretation of protein structures: Estimation of static accessibility: J. Mol. Biol. 55, 379-400 (1971)

23. Linderstrgm-LaNG, K.: On the ionization of proteins. Compt. Rend. Trav. Lab. Carlsberg 15 , $1-29$ (1924)

24. Linderstrom-Lang,K.: Proteins and enzymes. Lane Medical Lecturer. Stanford Univ. Publ. Med. Series, Vol. 6, 1-115 (1952)

25. Lumry, R. \& A. Rosenberg: The mobile defect hypothesis of protein function. Coll. Int. C. N. R. S. L'Eau. Syst. Biol. 246, 55-63 (1975)

26. Millero, F. J., G. K. Ward \& P. Chetirkim: Partial specific volume, expansibility, compressibility and heat capacity of aqueous lysozyme solutions. J. Biol. Chem. 251, 4001-4004 (1976)

27. Molday, R. S., S. W. Englander \& R. G. Kallen: Primary structure effects on peptide group hydrogen exchange. Biochemistry 11 , $150-158(1972)$

28. Nakanishi, M., M. Tsuboi \& A. Ikegami: Fluctuations of the myoglobin structure. Bull. Chem. Soc. Japan 47, 293-298 (1974)

29. Nedev, K. N., R. I. Volkova, Y. I. Khargin \& D. S. ChernavskiI: Modelling the structure of the protein globule. Bifizika 19, 983-986 (1974)

30. Nedev, K. N \& Y. I. Khurgin: Investigation of the surface layer of protein globules. Hydration of the $\alpha$-chymotrypsin molecule. Molekulyarnaya Biologiya 9, 761-767 (1975)

31. Peticolas, W. L.: Low frequency vibrations and the dynamics of proteins and polypeptides. Methods in Enzymology - Protein structure, in press (1979)

32. Reynolds, J. A., D. B. Gilbert \& C. Tanford: Empirical correlation between hydrophobic free energy and aqueous cavity surface area. Proc. Natl. Acad. Sci. USA 71, 2925-2927 (1974)

33. Richards, F. M.: The interpretation of protein structures: Total volume Group volume distributions and Packing density. J. Mol. Biol. 82, 1-14 (1974)

34. Richards, F. M.: Areas, volumes, packing and protein structure. Ann. Rev. Biophys. and Bioeng. 6, 151-176 (1977)

35. Richards, F. M., H. W. Wyckoff, W. D. Carlson, N. M. Allewell, N. Lee \& Y. Mitsui: Protein structure, ribonuclease-S and nucleotide interactions. Cold Spring Harbour Symp. Quant. Biol. XXXVI 35-43 (1971)

36. RichaRds, F. M. \& T. Richmond: Solvents, Interfaces and protein structure. Ciba sympo- 
sium No. 60, „Molecular Interactions and Activity in Proteins«, Excerpta Medica, Oxford, pp. 23-46 (1978)

37. Richardson, J.: The anatomy and Taxonomy of protein structure. Adv. Protein Chem. 33, in press (1979)

38. Richmond, T. J. \& F. M. Richards: Geometrical contraints and contact areas. J. Mol. Biol. 119, 537-555 (1978)

39. Rose, G. D.: Prediction of chain turns in globular proteins on a hydrophobic basis. Nature 272, 586-590 (1978)
40. Takano, T.: Structure of myoglobin refined at $2.0 \AA$ resolution. II. Structure of deoxymyoglobin from sperm whale. J. Biol. 110, 569-584 (1977)

41. Woodward, C. K. \& B. D. Hilton: Hydrogen exchange kinetics and internal motions in proteins and nucleic acids. Ann. Rev. Biophys. Bioeng., in press (1979)

42. WÜthrich, K. \& G. Wagner: Internal motion in globular proteins. Trends in biochemical sciences $3,227-230$ (1978) 